\title{
How palliative care teams can mitigate financial toxicity in cancer care
}

\author{
Ramy Sedhom ${ }^{1,2} \cdot$ Lindsey MacNabb $^{3} \cdot$ Thomas J. Smith $^{3} \cdot$ K. Robin Yabroff ${ }^{4}$
}

Received: 25 January 2021 / Accepted: 9 May 2021 / Published online: 14 May 2021

(c) The Author(s), under exclusive licence to Springer-Verlag GmbH Germany, part of Springer Nature 2021

\begin{abstract}
The disruption to patient and family well-being introduced by the rising costs of cancer care is a growing clinical problem. In addition to logistical questions, there is a compelling, existential one: "How should healthcare teams address patient and caregiver distress and uncertainty from financial toxicity?" We argue that the principles and practice of palliative care can help alleviate this element and often unaddressed component of human suffering.
\end{abstract}

Keywords Financial toxicity $\cdot$ Supportive care $\cdot$ Palliative care $\cdot$ Costs of care

Palliative care is defined by the World Health Organization as "an approach that improves the quality of life of patients (adults and children) and their families who are facing problems associated with life-threatening illness. It prevents and relieves suffering through the early identification, correct assessment and treatment of pain and other problems, whether physical, psychosocial or spiritual" [1]. Palliative care teams, usually comprising nurses, advance practice nurses, social workers, physicians, chaplains, and pharmacists, serve a seriously ill patient population dealing with some manner of anguish. Their success, as demonstrated by multiple randomized clinical trials, stems from the routine assessment of symptom burden, psychosocial and spiritual care needs, and individual values of the patients for whom they care [2]. The evidence from multiple randomized trials is so strong that the American Society of Clinical Oncology recommends that all advanced

Ramy Sedhom

sedhomr@mskcc.org

1 Department of Supportive Care, Memorial Sloan Kettering, New York, NY, USA

2 Supportive Care Service, Department of Medicine, Memorial Sloan Kettering Cancer Center, 1275 York Avenue, New York, NY 10065, USA

3 Palliative Care Program, Sidney Kimmel Comprehensive Cancer Center, Johns Hopkins University School of Medicine, Baltimore, MD, USA

4 Surveillance and Health Services Research, American Cancer Society, Atlanta, GA, USA cancer patients be seen by a multidisciplinary palliative care team within eight weeks of diagnosis [3].

Untapped to date is the opportunity for palliative care to assist in a growing component of patient sufferingfinancial toxicity. In recent years, due to increases in costsharing, through rising deductibles, co-insurance, and copayments, patients are shouldering larger proportions of healthcare costs [4]. The experience of financial toxicity has become both common and global [5, 6], with families experiencing a range of distress, and even personal bankruptcy [7]. An early and still appropriate example of financial toxicity is the impact of co-payments on the use of curative therapy for chronic myelogenous leukemia. People with higher co-pays were almost twice as likely to discontinue use of imatinib (GleevecTM) and had higher rates of non-adherence, leading to relapse and drug resistance [8]. Financial strain negatively impacts emotional well-being [9], health-related quality of life [10], treatment compliance [11], and even survival [12]. Cancer patients who filed for bankruptcy had an increased mortality risk compared to similar cancer patients who did not [12]. If financial toxicity was graded as an adverse event in a drug trial, toxicities would be high grade and dose limiting.

The evidence is scant regarding how to engage with patients in discussions about costs of cancer care, and many logistical questions remain $[13,14]$. What is known and has been particularly realized over this past year amidst global COVID-19 pandemic is the role of specialty palliative care teams to provide an extra layer of support. Our teams are present during situations of medical complexity and times of overwhelming uncertainty. In recent months, palliative 
care teams have filled gaps in care due to coronavirus by serving in acute care hospitals and long-term facilities. They have organized hotline telephone programs for distancebased conversations with patients and worried loved ones who could not physically see them, debriefing sessions for morally distressed healthcare providers, and participated in policy discussions regarding resource allocation and how to address disparities in care for vulnerable populations [15]. Palliative care team structures are unique, based on resources and funding. While palliative care teams are expanding (both within the USA and globally), availability is not always well defined. Importantly, national and international guidelines recommend involving palliative care for all patients with serious illness (regardless of site of care) [16-18]. These experiences highlight that the principles of palliative care are foundational to alleviate patient and system distress, and that is why the specialty is uniquely equipped to address financial toxicity.

The core principle of palliative care is to understand the whole patient - their life, their loved ones, and their medical and non-medical sources of strength and distress. We envision that these core principles would allow palliative care teams to rise to the present challenge in cancer care, where patients and families often remain unaware of the risk of financial toxicity, and how it can impact daily living, quality of life, and the ability to achieve personal and professional goals. Research in oncology continuously highlights gaps in what patients want to know-the required time away from home and frequency of clinic visits for different regimens, the associated costs of transportation, lodging, and caregiving arrangements, the costs of parking [19-21]. Addressing financial toxicity is well within the purview of palliative care, whose focus is on the entire family, seeking to improve the ability of families to support themselves through an often traumatic journey of cancer treatment.

It is troubling when data suggests that $50 \%$ of surveyed patients are willing to declare bankruptcy to pay for cancer care, and even more distressing when $70 \%$ of surveyed patients have late-stage disease, suggesting limited comprehension of the nonexistent gains such financial sacrifices tend to produce [7]. If third-line therapy for melanoma extends life by 3 months, but is accompanied by an outof-pocket cost of $\$ 100,000$, or foreclosure of one's home, would patients and families approach care decisions differently? Unfortunately, these theoretical choices are often a reality, though not discussed a priori.

Admittedly, out-of-pocket costs and financial toxicity are difficult to predict, but so are many medical outcomes that are nevertheless discussed daily in palliative care clinics, such as mortality chances that presage advance care planning. Although palliative care providers may not always have the answers to questions regarding costs of treatment or the ability to modify treatment recommendations, they are unique in their ability to coordinate care within teams and to prioritize treatment goals within a patient-centered framework. Nearly all palliative care teams ask patients about values, goals and fears, and the pertinent family dynamics before considering any clinical plan of action. These questions can be coordinated with medical oncology teams especially within the context of choosing appropriate therapy for patients with advanced cancer, a poor prognosis, and limited time. From a policy perspective, financial reimbursement could be considered to incentivize these conversations, similar to recent Medicare innovation to promote advance care planning [22]. Such discussions could assist patients who are willing to trade-off some chance of medical benefit for less financial strain.

In addition, having a liaison not directly involved with treatment decisions to discuss costs of care aligns with what patients want [23]. The majority of patients report a desire to discuss cost, and oncologists feel responsible to do so. Yet, most oncologists feel ill prepared to engage in these conversations [24]. In one study of metastatic patients with cancer, only $28 \%$ had discussed costs of care with their doctor [25]. When costs are not included in decision-making, patients and their families are ultimately the ones who suffer.

Palliative care teams have historically normalized difficult conversations. Expanding these conversations to include financial toxicity makes clinical sense, if we think of financial costs as treatment side effects and components of human suffering. In fact, screening for financial hardship could be considered part of broader efforts to assess the social determinants of health (e.g., food insecurity, housing instability), since patients may be making trade-offs between other needs and medical care [26]. There are actionable ways to normalize the discussion about costs and ability to pay for treatment. One of us (TJS) uses the simple question: "Are you have difficulty paying for your care?" The Cost Conversation project of Avalere Health and the Robert Wood Johnson Foundation also give practical tips [27], and there are published screening tools [26]. Interviews of patients suggest that the cost of care conversations should be held in a private place, with emotional support, and with someone who understands and can help [28]. When patients at risk are identified, medical oncologists and all members of the cancer care team should be alerted, and careful deliberation is made to provide patient-centered care that does not cause financial ruin.

The COVID-19 pandemic has also uniquely contributed to adverse economic effects, including widespread unemployment and loss of access to employer-sponsored health insurance, which can limit financial resources and increase risk of financial toxicity [4]. It has also limited in-person interactions with providers. Understanding the effects of the COVID-19 pandemic on cancer care, including any differences between virtual and in-person conversations about 
costs of care and financial hardship, will be important for future research.

Palliative care will always recognize the patient and family as a unit. Patients know their values, needs, preferences, and toxicity thresholds better than anyone else. A diagnosis of cancer is often overwhelming, and treatments can be toxic. What cancer should not be is financially catastrophic. It is time for palliative care teams to respond to this element of human suffering.

Authors' contributions All authors contributed equally to the planning and writing.

Funding Ramy Sedhom is supported by a Young Investigator Award Conquer Cancer Grant from the American Society of Clinical Oncology.

\section{Declarations}

Conflict of interest The authors declare no competing interests.

\section{References}

1. Palliative Care. https://www.who.int/news-room/fact-sheets/detail/ palliative-care. Accessed 15 Oct 2020

2. Bickel KE, McNiff K, Buss MK, Kamal A, Lupu D, Abernethy AP, Broder MS, Shapiro CL, Acheson AK, Malin J, Evans T, Krzyzanowska MK (2016) Defining high-quality palliative care in oncology practice: an american society of clinical oncology/ american academy of hospice and palliative medicine guidance statement. J Oncol Pract 12(9):e828-e838. https://doi.org/10. 1200/JOP.2016.010686

3. Ferrell BR et al (2017) Integration of palliative care into standard oncology care: American Society of Clinical Oncology Clinical Practice Guideline update. J Clin Oncol 35(1):96-112

4. Yabroff KR et al (2018) Medical financial hardship among cancer survivors in the United States: what do we know? What Do We Need to Know? Cancer Epidemiol Biomark Prev 27(12):1389-1397

5. Zafar SY (2015) Financial toxicity of cancer care: It's time to intervene. J Natl Cancer Inst 108(5):djv370. https://doi.org/10. 1093/jnci/djv370

6. Schilsky RL, Wehrwein P (2015) Gains against cancer, but enter 'financial toxicity. Manag Care 24(10):46-7-52-4

7. Chino $F$ et al (2018) Going for broke: a longitudinal study of patient-reported financial sacrifice in cancer care. J Oncol Pract 14(9):e533-e546

8. Dusetzina SB et al (2014) Cost sharing and adherence to tyrosine kinase inhibitors for patients with chronic myeloid leukemia. J Clin Oncol 32(4):306-311

9. Meeker CR et al (2016) Relationships among financial distress, emotional distress, and overall distress in insured patients with cancer. J Oncol Pract 12(7):e755-e764
10. Zafar SY et al (2015) Population-based assessment of cancer survivors' financial burden and quality of life: a prospective cohort study. J Oncol Pract 11(2): 145-150

11. Neugut AI et al (2011) Association between prescription copayment amount and compliance with adjuvant hormonal therapy in women with early-stage breast cancer. J Clin Oncol 29(18):2534-2542

12. Ramsey SD et al (2016) Financial insolvency as a risk factor for early mortality among patients with cancer. J Clin Oncol 34(9):980-986

13. Pisu M et al (2019) Perspectives on conversations about costs of cancer care of breast cancer survivors and cancer center staff a qualitative study. Ann Intern Med 170(9):S54-S38

14. Darien G et al (2020) Talking about the cost of care: a critical component of shared decision making patient and provider perspectives. J Natl Med Assoc 112(5):503-506

15. Kamal AH, Casarett DJ, Meier DE (2020) Compassion in a crisis: the role of palliative care during the COVID-19 pandemic. Mayo Clin Proc 95(11):2325-2326

16. Ferrell BR et al (2017) Integration of palliative care into standard oncology care: American Society of Clinical Oncology Clinical Practice Guideline update. J Clin Oncol 35(1):96-+

17. Cherny $\mathrm{N}$ et al (2010) European Society for Medical Oncology (ESMO) program for the integration of oncology and palliative care: a 5-year review of the Designated Centers' incentive program. Ann Oncol 21(2):362-369

18. Hui D et al (2015) Indicators of integration of oncology and palliative care programs: an international consensus. Ann Oncol 26(9):1953-1959

19. Chino F et al (2014) Self-reported financial burden and satisfaction with care among patients with cancer. Oncologist 19(4):414-420

20. Chino F et al (2017) Out-of-pocket costs, financial distress, and underinsurance in cancer care. JAMA Oncol 3(11):1582-1584

21. Premnath N, Grewal US, Gupta A (2020) Park the parking. JCO Oncol Pract 16(5):215-217

22. Weissman JS et al (2020) Recent trends in the use of edicare advance care planning codes. J Palliat Med 23(12):1568-1570

23. Alexander GC, Casalino LP, Meltzer DO (2003) Patient-physician communication about out-of-pocket costs. JAMA 290(7):953-958

24. Henrikson NB et al (2014) Patient and oncologist discussions about cancer care costs. Support Care Cancer 22(4):961-967

25. Kelly RJ et al (2015) Patients and physicians can discuss costs of cancer treatment in the clinic. J Oncol Pract 11(4):308-+

26. Yabroff KR, Bradley CJ, Shih YT (2021) Improving the process of screening for medical financial hardship in oncology practice. Cancer Epidemiol Biomarkers Prev 30(4):593-596

27. Actionable resources to facilitate cost conversations between patients and clinicians. https://avalere.com/insights/providingactionable-resources-to-facilitate-cost-of-care-conversationsbetween-patients-and-clinicians. Accessed 16 Oct 2020

28. Pisu $\mathrm{M}$ et al (2020) How, when, and with whom should cost of care conversations occur? Preferences of Two Distinct Cancer Survivor Groups. JCO Oncol Pract 16(9):594-+

Publisher's note Springer Nature remains neutral with regard to jurisdictional claims in published maps and institutional affiliations. 